\title{
Invertibility of nonsmooth mappings
}

\author{
Marcelo Montenegro and Adilson E. Presoto
}

\begin{abstract}
Let $F: \mathbb{R}^{N} \rightarrow \mathbb{R}^{N}$ be a locally Lipschitz continuous function. We prove that $F$ is a global homeomorphism or only injective, under suitable assumptions on the subdifferential $\partial F(x)$. We use variational methods, nonsmooth inverse function theorem and extensions of the Hadamard-Levy Theorem. We also address questions on the Markus-Yamabe conjecture.
\end{abstract}

\section{Introduction}

The start point is the following result due to Hadamard [18] and Palais [29] p. 129. A proof based on the Mountain Pass Lemma was given in Katriel [22].

Hadamard-Palais Theorem. Let $F: \mathbb{R}^{N} \rightarrow \mathbb{R}^{N}$ be a $C^{1}$ local diffeomorphism. If $\|F(x)\| \rightarrow \infty$ as $\|x\| \rightarrow \infty$, then $F$ is a global diffeomorphism.

The following theorem is known as the Hadarmad-Levy theorem, see Hadamard [18] and Levy [24]. A rigorous proof can be found in Plastock [31] and in Radulescu \& Radulescu [33].

Hadamard-Levy Theorem. Let $E$ and $G$ be two Banach spaces and $F: E \rightarrow G$ be a $C^{1}$ function such that $F^{\prime}(x)$ is invertible for each $x \in E$. If there exists a continuous map $\omega: \mathbb{R}_{+} \rightarrow \mathbb{R}$ such that

(i) $\int_{0}^{\infty} \frac{1}{\omega(t)} d t=\infty$;

(ii) $\left\|\left[F^{\prime}(x)\right]^{-1}\right\| \leq \omega(\|x\|)$.

Then $F$ is a $C^{1}$ global diffeomorphism.

One can easily see that for some positive constants $a, b$ the maps $\omega_{1}(t)=1$, $\omega_{2}(t)=a t+b, \omega_{3}(t)=(a t+b) \ln (t+2), \omega_{4}(t)=(a t+b) \ln (t+2) \ln \ln (t+3)$ satisfy the latter condition (i).

Key words and phrases: injectivity, invertibility, homeomorphism, Lipschitz continuous functions, Markus-Yamabe Conjecture.

2010 Mathematics Subject Classification: 26A16, 26B10, 37E30, 49J40, 49J52. 
We work with locally Lipschitz continuous functions $F: \mathbb{R}^{N} \rightarrow \mathbb{R}^{N}$ having conditions on the subdifferential $\partial F(x)$. In Section 2 we extend the Hadamard-Palais Theorem by means of variational methods due to Chang [7] and inverse function theorem of Clarke [10] and [11]. In Section 3 we show a relationship between Markus-Yamabe Conjecture and results from [5], [6], [14] and [16], by means of another extension of the Hadamard-Palais Theorem due to Pourciau [32].

Markus-Yamabe Conjecture. Let $F: \mathbb{R}^{N} \rightarrow \mathbb{R}^{N}$ be a $C^{1}$ function such that $F(0)=0$. If for every $x \in \mathbb{R}^{N}$ all eigenvalues of $F^{\prime}(x)$ have negative real parts, then 0 is a global attractor of $x^{\prime}=F(x)$.

It is well known that 0 is a local attractor of $x^{\prime}=F(x)$. The conjecture is true for $N=2$, and it is false for $N \geq 3$, see [15].

A series of results related to the Markus-Yamambe Conjecture and, particularly, to one of its closest results, the Jacobian conjecture, was established in the last decades. An attempt to prove the latter conjecture have guided Chamberland and Meisters to the following theorem, see [6] and [30].

Theorem of Chamberland and Meisters. Let $F: \mathbb{R}^{N} \rightarrow \mathbb{R}^{N}$ be a $C^{1}$ function with the property that there is an $\varepsilon>0$ such that $|\mu| \geq \varepsilon$ for every eigenvalue $\mu$ of $F^{\prime}(x) F^{\prime}(x)^{T}$ for every $x \in \mathbb{R}^{N}$, then $F$ is injective (superscript $T$ stands for transposition of matrices).

A weak version of the Markus-Yamabe conjecture has been stated in [16].

Weak Markus-Yamabe Conjecture. Let $F: \mathbb{R}^{N} \rightarrow \mathbb{R}^{N}$ be a $C^{1}$ function. If all eigenvalues of $F^{\prime}(x)$ for every $x \in \mathbb{R}^{N}$ have negative real part, then $F$ is injective.

In [16] it has been proved that the weak Markus-Yamabe conjecture holds in the case that the function $F$ is $C^{1}$ Lipschitz.

Another related conjecture due to Alexandrov [1] stated later in [6] reads as follows, see also [2].

Alexandrov-Chamberland-Meisters Conjecture. Let $F: \mathbb{R}^{N} \rightarrow \mathbb{R}^{N}$ be a $C^{1}$ function. If there exists $\varepsilon>0$ such that for every $x \in \mathbb{R}^{N}$ all eigenvalues $\mu$ of $F^{\prime}(x)$ satisfy $|\mu| \geq \varepsilon$, then $F$ is injective.

In [6], the authors have used a reduction-of-degree argument to show that if the above conjecture is true, then it implies that the Jacobian conjecture is also true. Furthermore, Biasi, Gutierrez and dos Santos in [5] have proved the validity of the Chamberland-Meisters Conjecture for $C^{1}$ Lipschitz functions. In Section 3 we shall prove a similar fact in the context of locally Lipschitz functions.

For more results on subdifferentials, injectivity, invertibility, diffeomorphisms and related subjects to the Markus-Yamabe conjecture we quote the papers [4], [8], [9], [12], [17], [19]-[21], [25], [27], [28], [34], [36], [37] and [38]. 


\section{Invertibility by means of Clarke's and Chang's theorems}

In this section we use variational methods combined with the inverse function theorem. We start by defining and writing the ingredients that will be useful to prove Theorem 2.1 below and its consequences.

Let $U \subset \mathbb{R}^{N}$ be an open set. A function $F: U \rightarrow \mathbb{R}^{N}$ is locally Lipschitz continuous if for every $x \in U$ there exists a constant $C>0$ and a neighborhood $V$ of $x$, $V \subset U$ such that $\|F(y)-F(z)\| \leq C\|y-z\|$ for every $y, z \in V$. When the inequality is true for every point of $U$, we say simply that $F$ is Lipschitz continuous.

Definition. The subdifferential introduced by Clarke [10] of a function $F: U \rightarrow$ $\mathbb{R}^{N}$ which is locally Lipschitz continuous is denoted by $\partial F(x)$ and defined by

$$
\partial F(x)=\bigcap_{\delta>0} \overline{\operatorname{conv}\left\{F^{\prime}(z): z \in U F^{\prime}(z) \text { exists }|z-x|<\delta\right\}},
$$

where $\operatorname{conv}\{A\}$ stands for the convex hull of the set $A$ and $\overline{\operatorname{conv}\{A\}}$ is the closure of the set $\operatorname{conv}\{A\}$. If all matrices in $\partial F(x)$ are nonsingular, we say that the subdifferential $\partial F(x)$ is invertible.

The classical local Inverse Function Theorem extends naturally to the locally Lipschitz continuous functions, see Clarke [11].

Clarke's Inverse Function Theorem. Let $U \subset \mathbb{R}^{N}$ be an open set and let $F: U \rightarrow \mathbb{R}^{N}$ be a locally Lipschitz continuous function. Let $x_{0} \in U$. If $\partial F\left(x_{0}\right)$ is invertible, then there exist open sets $V, W \subset \mathbb{R}^{N}$ such that $x_{0} \in V \subset U, F\left(x_{0}\right) \in W$ and $F: V \rightarrow W$ is a homeomorphism. Moreover, $F^{-1}$ is locally Lipschitz.

We will use several times the chain rule provided in Clarke, [11, Lemma 2].

Chain Rule Lemma. Let $g: \mathbb{R}^{N} \rightarrow \mathbb{R}$ be a $C^{1}$ function and assume that $h$ : $\mathbb{R}^{N} \rightarrow \mathbb{R}^{N}$ is Lipschitz continuous on a neighborhood of $x \in \mathbb{R}^{N}$. Then $\partial(g \circ h)(x) \subset$ $g^{\prime}(h(x)) \partial h(x)$.

We will use the definition below given in [7] to deal with nonsmooth functionals.

Definition. Let $f: \mathbb{R}^{N} \rightarrow \mathbb{R}$ be a locally Lipschitz continuous function and let $\lambda: \mathbb{R}^{N} \rightarrow \mathbb{R}$ be the function defined by $\lambda(x)=\inf \left\{\left\|x^{*}\right\|: x^{*} \in \partial f(x)\right\}$. The $\underline{\text { Palais-Smale }}$ condition for $f$ at level $c$ means that every sequence $\left(x_{n}\right)$ in $\mathbb{R}^{N}$ such that $f\left(x_{n}\right) \rightarrow c$ and $\lambda\left(x_{n}\right) \rightarrow 0$ admits a convergent subsequence, where $\lambda\left(x_{n}\right)=\inf \left\{\left\|x^{*}\right\|: x^{*} \in\right.$ $\left.\partial f\left(x_{n}\right)\right\}$. When the property holds for every level $c$, we simply say that $f$ satisfies the Palais-Smale condition. The sequence $\left(x_{n}\right)$ is called Palais-Smale sequence.

The next result establishes the existence of the Palais-Smale sequences, for a proof by means of Deformation Lemma, see [7]. 
Chang's Theorem. Let $f: \mathbb{R}^{N} \rightarrow \mathbb{R}$ be a locally Lipschitz continuous function. If there exist $x_{0}, x_{1} \in \mathbb{R}^{N}$ and an open set $\Omega \subset \mathbb{R}^{N}$ satisfying $\max \left\{f\left(x_{0}\right), f\left(x_{1}\right)\right\}<$ $\inf _{x \in \partial \Omega} f(x)$, then there exists a sequence $\left(x_{n}\right)$ in $\mathbb{R}^{N}$ such that $f\left(x_{n}\right) \rightarrow c$ and $\lambda\left(x_{n}\right) \rightarrow 0$, with

$$
c=\inf _{\gamma \in \Gamma} \max _{t \in[0,1]} f(\gamma(t)) \quad \text { and } \quad \Gamma=\left\{\gamma \in C^{0}\left([0,1], \mathbb{R}^{N}\right): \gamma(0)=x_{0} \text { and } \gamma(1)=x_{1}\right\}
$$

We state and prove next one of the main theorems of this section, which can be viewed as a generalization of the Hadamard-Levy Theorem, since coercivity implies the Palais-Smale condition, we return to this idea in the corollaries.

Theorem 2.1. Let $F: \mathbb{R}^{N} \rightarrow \mathbb{R}^{N}$ be a locally Lipschitz continuous function such that $\partial F(x)$ is invertible for every $x \in \mathbb{R}^{N}$. If $|F|$ satisfies the Palais-Smale condition, then $F$ is injective.

Proof of Theorem 2.1. We first note that by [23, Corollary 3], $|F|$ is coercive. According to the Clarke's Inverse Function Theorem, $F$ is a local homeomorphism. Suppose on the contrary that $F$ is not injective, thus there exist $a_{1}, a_{2} \in \mathbb{R}^{N}, a_{1} \neq a_{2}$, such that $F\left(a_{1}\right)=F\left(a_{2}\right)$. Let $y=F\left(a_{1}\right)=F\left(a_{2}\right)$. Define $f(x)=|y-F(x)|^{2} / 2$.

Since $F$ is a local homeomorphism, $a_{1}$ and $a_{2}$ are strict local minima of $f$. From the Chain Rule Lemma, it follows that

$$
\partial f(x) \subset(F(x)-y)^{T} \partial F(x) \text { for every } x \in \mathbb{R}^{N},
$$

where the superscript $T$ stands for transposition of matrices.

The function $f$ satisfies Palais-Smale condition at positive levels. Indeed if $\left(x_{n}\right)$ is a sequence satisfying $f\left(x_{n}\right) \rightarrow c>0$ and $\lambda\left(x_{n}\right) \rightarrow 0$, by the coercivity of $|F|$ we have $\left(x_{n}\right)$ is bounded, so that it has a subsequence converging to a point $x \in \mathbb{R}^{N}$. Since $\lambda$ is lower semi-continuous we have $\lambda(x)=0$.

We apply now Chang's Theorem to obtain a sequence $\left(x_{n}\right)$ in $\mathbb{R}^{N}$ satisfying $f\left(x_{n}\right) \rightarrow c$ and $\lambda\left(x_{n}\right) \rightarrow 0$, where $c>0$. In view of the Palais-Smale condition for $f$, for a subsequence, without loss of generality relabelled as $\left(x_{n}\right)$, one obtains $x_{n} \rightarrow \tilde{x}$.

By (1) we conclude that $0 \in(y-F(\tilde{x}))^{T} \partial F(\tilde{x})$, i.e., there exists a matrix $M \in$ $\partial F(\tilde{x})$ such that $0=(y-F(\tilde{x}))^{T} M$. However, since $f(\tilde{x})>0$, we obtain $y-F(\tilde{x}) \neq 0$. Therefore $M$ is not invertible, which is a contradiction.

Example. The hypotheses of Theorem 2.1 do not guarantee the global inversion of $F$. The example $F: \mathbb{R}^{2} \rightarrow \mathbb{R}^{2}$,

$$
F(x, y)=\left(|x|+y\left(1+x^{2}\right),|y|+2 \arctan (x)\right),
$$


from [11], furnishes a function with invertible subdifferential $\partial F(x, y)$ at every point $(x, y) \in \mathbb{R}^{2}$. Indeed, for points where $F$ is nondifferentiable, say, if $y \neq 0$, we have

$$
\partial F(0, y)=\left\{\left[\begin{array}{cc}
s & 1 \\
2 & \operatorname{sign}(y)
\end{array}\right]:-1 \leq s \leq 1\right\},
$$

which elements are non-singular matrices. In a similar way, on the line $y=0, x \neq 0$ we obtain non-singular matrices

$$
\partial F(x, 0)=\left\{\left[\begin{array}{cc}
\operatorname{sign}(x) & 1+x^{2} \\
\frac{2}{1+x^{2}} & t
\end{array}\right]:-1 \leq t \leq 1\right\} .
$$

At the origin,

$$
\partial F(0,0)=\left\{\left[\begin{array}{ll}
s & 1 \\
2 & t
\end{array}\right]:-1 \leq s \leq 1,-1 \leq t \leq 1\right\},
$$

which elements also have nontrivial determinants. Otherwise, one may verify that the half-space

$$
S=\left\{(x, y) \in \mathbb{R}^{2}: y \leq-\pi\right\}
$$

is contained in the complement of the image of $F$, that is, every $(a, b) \in S$ has no preimage, hence $F$ is not surjective.

As we have said before, since coercivity implies Palais-Smale condition, one obtains the next global injection result, global homemorphism in reality, which resembles the Hadamard-Palais Theorem.

Corollary 2.2. Let $F: \mathbb{R}^{N} \rightarrow \mathbb{R}^{N}$ be a locally Lipschitz continuous function such that $\partial F(x)$ is invertible for every $x \in \mathbb{R}^{N}$. If $|F|$ is coercive, which means that $|F(x)| \rightarrow \infty$ as $|x| \rightarrow \infty$, then $F$ is a global homemorphism.

Proof of Corollary 2.2. The coercivity implies the Palais-Smale condition for $|F|$, a contradiction reasoning explains that. Therefore, $F$ is injective by Theorem 2.1. Moreover, the set $F\left(\mathbb{R}^{N}\right)$, is closed. And by Clarke's Inverse Function Theorem $F\left(\mathbb{R}^{N}\right)$ is also open. By connectness, $F$ is surjective, thus it is a global homemorphism.

A function $F: \mathbb{R}^{N} \rightarrow \mathbb{R}^{N}$ is proper if the preimage of compact sets is a compact set. Under the continuity assumption of the preceding Corollary, coercivity and properness are equivalent, implying the Palais-Smale condition for $|F|$. Therefore, properness implies global injection, and we regain the Banach-Mazur criterion for global homemorphism [3]. 
We state below a particular result that works for $C^{1}$ functions, see Katriel [22] for a proof.

Corollary 2.3. If $F: \mathbb{R}^{N} \rightarrow \mathbb{R}^{N}$ is a $C^{1}$ function, such that $|F|$ satisfies the Palais-Smale condition and $F^{\prime}(x)$ is an isomorphism for every $x \in \mathbb{R}^{N}$, then $F$ is injective.

In the sequel we state another injectivity theorem for nonsmooth mappings. Compare with the Theorem of Chamberland and Meisters we have stated in Section 1 , consult [6] for more details.

Theorem 2.4. Let $F: \mathbb{R}^{N} \rightarrow \mathbb{R}^{N}$ be a locally Lipschitz continuous function such that $\partial F(x)$ is invertible for every $x \in \mathbb{R}^{N}$. Suppose that there is an $\varepsilon>0$ and $K>0$ such that for every $M \in \partial F(x)$ and $|x| \geq K$ the eigenvalues $\mu$ of $M M^{T}$ satisfy $|\mu| \geq \varepsilon$, then $F$ is injective.

Proof of Theorem 2.4. Suppose that $F$ is not injective, hence there exist $a_{1}, a_{2} \in \mathbb{R}^{N}, a_{1} \neq a_{2}$, such that $F\left(a_{1}\right)=F\left(a_{2}\right)$. Define $G(x)=F\left(a_{1}+a_{2}-x\right)-F\left(a_{1}\right)$, thus $G\left(a_{1}\right)=G\left(a_{2}\right)=0$. Define the function

$$
f(x)=\frac{1}{2}|G(x)|^{2} .
$$

By Clarke's Inverse Function Theorem, $F$ is a local homeomorphism, then $a_{1}$ and $a_{2}$ are isolated zeros of $f$. By virtue of Chang's Theorem there exists a sequence $\left(x_{n}\right)$ satisfying $f\left(x_{n}\right) \rightarrow c$ and $\lambda\left(x_{n}\right) \rightarrow 0$, where $c>0$.

Assume by contradiction that $f$ satisfies the Palais-Smale condition at level $c$. Thus, there exists a subsequence of $\left(x_{n}\right)$, still denoted by $\left(x_{n}\right)$, satisfying $x_{n} \rightarrow x$. In view of the lower-semicontinuity of $\lambda$, we conclude that $\lambda(x)=0$. Since $\partial f(x)$ is compact, see [35, Theorem 17.2], $0 \in \partial f(x) \subset G(x)^{T} \partial G(x)$. By hypothesis $\partial G(x)$ contains only invertible matrices, so that $f(x)=\frac{1}{2}|G(x)|^{2}=0$. On the other hand $f(x)=c>0$, which is a contradiction. Therefore $\left(x_{n}\right)$ is not a Palais-Smale sequence. Thus there is a subsequence, still denoted by $\left(x_{n}\right)$, such that $\left|x_{n}\right| \rightarrow \infty$. In particular, $\left|x_{n}\right| \geq K$ for large enough $n$. Notice that if the subsequence $\left(x_{n}\right)$ were bounded, then there would exist a convergent subsequence, contradicting the fact that $\left(x_{n}\right)$ is not a Palais-Smale sequence.

The set of subgradients at each point is compact. Hence for each $x_{n}$ there exists a matrix $M_{n}=M_{n}\left(x_{n}\right)$, i.e. depending on the point $x_{n}$, such that $\lambda\left(x_{n}\right)=$ $\left\|G\left(x_{n}\right)^{T} M_{n}\right\|$. The variational characterization of the first eigenvalue of a symmetric matrix $A$ is given by the formula

$$
\mu=\inf _{y \in \mathbb{R}^{N}, y \neq 0} \frac{\langle A y, y\rangle}{|y|^{2}} .
$$


Taking $A=M_{n} M_{n}^{T}$ and denoting by $\mu_{n}$ its first eigenvalue (with respect to $x_{n}$ ) we get

$$
0<\varepsilon \leq \mu_{n} \leq \frac{G\left(x_{n}\right)^{T} M_{n} M_{n}^{T} G\left(x_{n}\right)}{G\left(x_{n}\right)^{T} G\left(x_{n}\right)}=\frac{\left\|G\left(x_{n}\right)^{T} M_{n}\right\|^{2}}{2 f\left(x_{n}\right)}=\frac{\lambda\left(x_{n}\right)}{2 f\left(x_{n}\right)} \longrightarrow 0
$$

which is a contradiction.

Example. It is worth to mention that the hypotheses of Theorem 2.4 can not be weakened to allow merely $\mu \neq 0$. As it can be seen by the example of the non-injective function $F(x, y)=\left(e^{x} \cos y, e^{x} \sin y\right)$, with positive eigenvalues of $F^{\prime}(x, y) F^{\prime}(x, y)^{T}$ equal to $e^{2 x}$, which converge to 0 as $x \rightarrow-\infty$. Furthermore the inverse function theorem for a $C^{1}$ function $F: \mathbb{R}^{N} \rightarrow \mathbb{R}^{N}$ says that $F$ is a local diffeomorphism if and only if $F^{\prime}(x)$ is invertible for every $x \in \mathbb{R}^{N}$. In dimension $N=1$ the invertibility of $F^{\prime}(x)$ implies the injectivity of $F$, since the function $F$ must be strictly increasing or decreasing. In higher dimensions, the invertibility of $F^{\prime}(x)$ no longer implies injectivity, as it can be seen by the standard above counter-example $F(x, y)=\left(e^{x} \cos y, e^{x} \sin y\right)$.

\section{Invertibility by means of the generalized Hadamard-Palais theorem}

In this section we use an extension of the Hadamard-Palais Theorem. Let $F: \mathbb{R}^{N} \rightarrow \mathbb{R}^{N}$ be a locally Lipschitz continuous function and $\partial F(x)$ its subdifferential. As in Pourciau [32] we define the following co-norms [.] of real matrices and of subdifferentials, namely

$$
[M]=\inf _{v \in \mathbb{R}^{N},\|v\|=1}\|M v\| \quad \text { and } \quad[\partial F(x)]=\inf _{M \in \partial F(x)}[M]
$$

Pourciau's Theorem. Let $F: \mathbb{R}^{N} \rightarrow \mathbb{R}^{N}$ be a locally Lipsichitz continuous function with $\partial F(x)$ is invertible for every $x \in \mathbb{R}^{N}$ and such that $m(t)=$ $\inf _{x \in \mathbb{R}^{N},\|x\| \leq t}[\partial F(x)]$ satisfies $\int_{0}^{\infty} m(t) d t=\infty$, then $F$ is a global homeomorphism.

In the following, we shall prove the Nonsmooth Chamberland-Meisters Conjecture under assumption that $F$ is Lipschitz continuous.

Theorem 3.1. Let $F: \mathbb{R}^{N} \rightarrow \mathbb{R}^{N}$ be a locally Lipschitz continuous function. Suppose that there is an $\varepsilon>0$ such that $|\mu| \geq \varepsilon$ for all eigenvalues $\mu$ of every $M \in$ $\partial F(x)$ and $x \in \mathbb{R}^{N}$. If there exists a constant $K>0$ such that $\|M\| \leq K$ for every $M \in \partial F(x)$ and $x \in \mathbb{R}^{N}$, then $F$ is a global homeomorphism. 
Proof of Theorem 3.1. By Dunford \& Schwartz [13, page 1020], if $L: \mathbb{R}^{N} \rightarrow \mathbb{R}^{N}$ is an invertible linear operator we have

$\left|\operatorname{det} L\left\|\left\langle L^{-1} y, z\right\rangle \mid \leq c_{N}\right\| y\|\| z\|\| L \|^{N-1}, \quad \forall y, z \in \mathbb{R}^{N}\right.$, where $c_{N}=(N-1)^{\frac{-(N-1)}{2}}$

Since $\partial F(x)$ is invertible, taking $y \in \mathbb{R}^{N}$ with $\|y\|=1, M \in \partial F(x), L=M$ and $z=$ $M^{-1} y$ in the above inequality we obtain

$$
|\operatorname{det} M|\left\|M^{-1} y\right\|^{2} \leq c_{N}\left\|M^{-1} y\right\|\|M\|^{N-1} .
$$

In view of the Jordan normal form, we have $|\operatorname{det} M| \geq \varepsilon^{N}$, thus

$$
\varepsilon^{N}\left\|M^{-1} y\right\|^{2} \leq c_{N}\left\|M^{-1} y\right\| K^{N-1}
$$

It follows that $\left\|M^{-1}\right\| \leq C$, hence $[M] \geq 1 / C$, where the constant $C>0$ is independent on $M$ and on $x \in \mathbb{R}^{N}$. The result follows from Pourciau's Theorem.

Notice that whenever $F: \mathbb{R}^{N} \rightarrow \mathbb{R}^{N}$ is Lipschitz continuous (not only locally), all matrices $M$ belonging to $\partial F(x)$ have bounded norm $\|M\| \leq R$, where $R>0$ is the Lipschitz constant of $F$. The next result is an immediate consequence of this remark.

Corollary 3.2. Let $F: \mathbb{R}^{N} \rightarrow \mathbb{R}^{N}$ be a Lipschitz continuous function. Suppose that there is an $\varepsilon>0$ such that $|\mu| \geq \varepsilon$ for all eigenvalues $\mu$ of every $M \in \partial F(x)$ and $x \in \mathbb{R}^{N}$, then $F$ is a global homeomorphism.

A slightly more general assumption on the boundedness of the subdifferential still permits us to obtain a global homeomorphism.

Proposition 3.3. Let $F: \mathbb{R}^{N} \rightarrow \mathbb{R}^{N}$ be a locally Lipschitz continuous function. Suppose that there is $\varepsilon>0$ such that $|\mu| \geq \varepsilon$ for all eigenvalues $\mu$ of every $M \in \partial F(x)$ and $x \in \mathbb{R}^{N}$. If there exists a constant $K>0$ such that $\|M\|\left\|M^{-1}\right\| \leq K$ for every $M \in \partial F(x)$ and $x \in \mathbb{R}^{N}$, then $F$ is a global homeomorphism.

Proof of Proposition 3.3. We now apply (2) with $L=M, y=z=x$ and $\|x\|=1$ to get

$$
\left|\operatorname{det} M\left\|\left\langle M^{-1} x, x\right\rangle \mid \leq c_{N}\right\| M\left\|^{N-1} \leq c_{N} K^{N-1}\right\| M^{-1} \|^{1-N} .\right.
$$

Since $|\operatorname{det} M| \geq \varepsilon^{N}$, thus

$$
\left\|M^{-1}\right\|=\sup _{x \in \mathbb{R}^{N}\|x\|=1}\left|\left\langle M^{-1} x, x\right\rangle\right| \leq\left(c_{N} K^{N-1}\right)^{1 / N} \leq C .
$$

As in the proof of Theorem 3.1, we conclude that $[M] \geq 1 / C$ for every $M \in \partial F(x)$ and $x \in \mathbb{R}^{N}$, where the constant $C$ is independent on $M$ and $x \in \mathbb{R}^{N}$. The conclusion follows from Pourciau's Theorem. 
For $C^{1}$ functions we recover two results obtained originally in Plastock [31].

Corollary 3.4. Let $F: \mathbb{R}^{N} \rightarrow \mathbb{R}^{N}$ be a $C^{1}$ function. If there exist $\varepsilon>0$ and $K>0$ such that $\left|\operatorname{det} F^{\prime}(x)\right| \geq \varepsilon$ and $\left\|F^{\prime}(x)\right\| \leq K$ for every $x \in \mathbb{R}^{N}$, then $F$ is a global diffeomorphism.

Corollary 3.5. Let $F: \mathbb{R}^{N} \rightarrow \mathbb{R}^{N}$ be a $C^{1}$ function. If there exist $\varepsilon>0$ and $K>0$ such that $\left|\operatorname{det} F^{\prime}(x)\right| \geq \varepsilon$ and $\left\|F^{\prime}(x)\right\|\left\|F^{\prime}(x)^{-1}\right\| \leq K$ for every $x \in \mathbb{R}^{N}$, then $F$ is a global diffeomorphism.

Example. The assumptions of Theorem 3.1 are essential. For instance, $\arctan x$ is Lipschitz continuous with constant 1 and $\arctan ^{\prime} x=1 /\left(1+x^{2}\right)>0$, but $\arctan x$ is not surjective. Another example is the $C^{1}$ function $F: \mathbb{R}^{2} \rightarrow \mathbb{R}^{2}$ defined by $F(x, y)=$ $\left(\arctan x, y\left(1+x^{2}\right)\right)$. Hence

$$
F^{\prime}(x, y)=\left[\begin{array}{cc}
\frac{1}{1+x^{2}} & 0 \\
2 x y & 1+x^{2}
\end{array}\right]
$$

has determinant equals to 1 . However, the image of $F$ is contained in the strip

$$
\left\{(a, b) \in \mathbb{R}^{2}:-\frac{\pi}{2}<a<\frac{\pi}{2}\right\} .
$$

Moreover, the eigenvalues of $F^{\prime}(x, y)$ and of $F^{\prime}(x, y) F^{\prime}(x, y)^{T}$ are not bounded away from the origin.

The Nonsmooth Weak Markus Yamabe Conjecture which holds for Lipschitz continuous functions reads as follows.

Theorem 3.6. Let $F: \mathbb{R}^{N} \rightarrow \mathbb{R}^{N}$ be a Lipschitz continuous function. If all eigenvalues of every $M \in \partial F(x)$ and for every $x \in \mathbb{R}^{N}$ have negative real part, then $F$ is injective.

Before proving the above result, we need to establish in the nonsmooth setting a technical lemma already proved by Biasi, Gutierrez and dos Santos [5] for $C^{1}$ functions.

Lemma 3.7. Let $F: \mathbb{R}^{N} \rightarrow \mathbb{R}^{N}$ be a locally Lipschitz continuous function such that $\partial F(x)$ is invertible for every $x \in \mathbb{R}^{N}$. If there exists a sequence $\left(t_{n}\right)$ of real numbers converging to zero and such that for every $n \in \mathbb{N}, F_{t_{n}}: \mathbb{R}^{N} \rightarrow \mathbb{R}^{N}$ defined by $F_{t_{n}}(x)=F(x)-t_{n} x$ is injective, then $F$ is injective.

Proof of Lemma 3.7. Suppose by contradiction that $F$ is not injective. Then there exist $a_{1}, a_{2} \in \mathbb{R}^{N}$ such that $a_{1} \neq a_{2}$ and $F\left(a_{1}\right)=F\left(a_{2}\right)$. By Clarke's Inverse Function Theorem, there are open sets $U_{1}$ and $U_{2}$, which we can suppose to be 
disjoint and such that $a_{1} \in U_{1}, a_{2} \in U_{2}$, such that the restrictions $F_{\left.\right|_{U_{1}}}$ and $F_{\left.\right|_{U_{2}}}$ are homeomorphisms. We now take $n$ large enough such that $y \in F_{t_{n}}\left(U_{1}\right) \cap F_{t_{n}}\left(U_{2}\right)$, where $y=F\left(a_{1}\right)=F\left(a_{2}\right)$. Hence there is an open set $V$ containing $y$ such that $V \subset\left(F_{t_{n}}\left(U_{1}\right) \cap F_{t_{n}}\left(U_{2}\right)\right)$. For points $z \in V$, there exist $z_{1} \in U_{1}$ and $z_{2} \in U_{2}$ satisfying $F_{t_{n}}\left(z_{1}\right)=F_{t_{n}}\left(z_{2}\right)=z$, which contradicts the injectivity of $F_{t_{n}}$.

Proof of Theorem 3.6. Let $\left(t_{n}\right)$ be a sequence of positive real numbers converging to zero. Consider the functions $F_{t_{n}}(x)=F(x)-t_{n} x, x \in \mathbb{R}^{N}$. Note that $\partial F_{t_{n}}(x)=\partial F(x)-t_{n} I$ and that the real part of the eigenvalues of the matrices in $\partial F_{t_{n}}\left(x_{n}\right)$ are less than $-t_{n}$. Moreover, for every eigenvalue $\mu$ of a matrix $M \in \partial F_{t_{n}}(x)$, we have $|\mu| \geq|\mathbb{R} e(\mu)| \geq\left|t_{n}\right|>0$. Here $\mathbb{R} e(\mu)$ denotes the real part of $\mu$. From Corollary 3.2, it follows that $F_{t_{n}}$ is a global homeomorphism. By Lemma 3.7, we conclude that $F$ is injective.

The Nonsmooth Chamberland-Meisters Conjecture implies the Nonsmooth Weak Markus-Yamabe Conjecture.

Theorem 3.8. The validity of Theorem 3.1 implies the validity Theorem 3.6.

Proof of Theorem 3.8. Let $K<0$ and $F: \mathbb{R}^{N} \rightarrow \mathbb{R}^{N}$ be such that the eigenvalues $\mu$ of $M \in \partial F(x), x \in \mathbb{R}^{N}$ satisfy $\mathbb{R} e(\mu)<K$, then $|\mu| \geq|\mathbb{R} e(\mu)| \geq|K|$, hence $F$ is injective. Here $\mathbb{R} e(\mu)$ denotes the real part of $\mu$. Therefore Theorem 3.6 is also verified.

Acknowledgement. M. Montenegro has been supported by CNPq and A. Presoto by FAPESP. The authors thank the referee for a number of valuable suggestions and references.

\section{References}

1. Alexandrov, V. A., Remarks on Efimov's theorem about differential tests of homeomorphism, Rev. Roumaine Math. Pures Appl. 36 (1991), 101-105.

2. Alexandrov, V. A., On a differential test of homeomorphism, found by N.V. Efimov, Contemporary Problems of Mathematics and Mechanics (Sovremennye Problemy Matematiki i Mekhaniki) 6 (2011), 18-26. arxiv:1010.3637, Moscow State University

3. Banach, S. and Mazur, S., Über mehrdeutige stetige abbildungen, Studia Math. 5 (1934), 174-178.

4. Benoist, J. and Hiriart-Urruty, J.-B., What is the subdifferential of the closed convex hull of a function? SIAM J. Math. Anal. 27 (1996), 1661-1679.

5. Biasi, C., Gutiérrez, C. and dos Santos, E. L., Global inverse mapping theorems, Cadernos de Matemática ICMC-USP 10 (2009), 9-18. 
6. Chamberland, M. and Meisters, G., A mountain pass to the Jacobian conjecture, Canad. Math. Bull. 41 (1998), 442-451.

7. Chang, K. C., Variational methods for nondifferentiable functionals and their applications to partial differential equations, J. Math. Anal. Appl. 80 (1981), $102-129$.

8. Cheban, D., Markus-Yamabe conjecture for non-autonomous dynamical systems, Nonlinear Anal. 95 (2014), 202-218.

9. Cima, A., Gasull, A. and Mañosas, F., Examples and counterexamples for MarkusYamabe and LaSalle global asymptotic stability problems, in Proceedings of the International Workshop Future Directions in Difference Equations, pp. 89-96, 2011.

10. Clarke, F. H., Generalized gradients and applications, Trans. Amer. Math. Soc. 205 (1975), 247-262.

11. Clarke, F. H., On the inverse function theorem, Pacific J. Math. 64 (1976), 97-102.

12. Cristea, M., Local inversion theorems without assuming continuous differentiability, J. Math. Anal. Appl. 143 (1989), 259-263.

13. Dunford, N. and Schwartz, J. T., Linear Operators. Part I, Wiley Classics Library, John Wiley \& Sons, New York, 1958.

14. Fernandes, A. and Gutiérrez, C., On local diffeomorphisms of $\mathbb{R}^{n}$ that are injective, Cadernos de Matemática ICMC-USP 1 (2002), 325-334.

15. Fernandes, A., Gutierrez, C. and Rabanal, R., Global asymptotic stability for differentiable vector fields of $R^{2}$, J. Differential Equations 206 (2004), 470-482.

16. Fernandes, A., Gutiérrez, C. and Rabanal, R., On local diffeomorphisms of $\mathbb{R}^{N}$ that are injective, Qual. Theory Dyn. Syst. 4 (2004), 255-262.

17. Gorni, G., A criterion of invertibility in the large for local diffeomorphisms between Banach spaces, Nonlinear Anal. 21 (1993), 43-47.

18. Hadamard, J., Sur les transformations ponctuelles, Bull. Soc. Math. France 34 (1906), 71-84.

19. Hernandez, J. E. and Nashed, M. Z., Global invertibility of expanding maps, Proc. Amer. Math. Soc. 116 (1992), 285-291.

20. Ioffe, A. D., Global surjection and global inverse mapping theorems in Banach spaces. Reports from the Moscow refusnik seminar, Ann. N.Y. Acad. Sci. 491 (1987), 181-188.

21. John, F., On the quasi-isometric mappings, Comm. Pure Appl. Math. 21 (1968), $77-110$.

22. Katriel, G., Mountain pass theorems and global homeomorphism theorems, Ann. Inst. H. Poincaré Anal. Non Linéaire 11 (1994), 189-209.

23. Kourogenis, N. C. and Papageorgiou, N. S., A weak nonsmooth Palais-Smale condition and coercivity, Rend. Circ. Mat. Palermo (2) 49 (2000), 521-526.

24. Levy, P., Sur les fonctions des lignes implicites, Bull. Soc. Math. France 48 (1920), $13-27$.

25. Li, H.-X., Liang, J. and XiaO, T.-J., Criteria for global invertibility of $\mathcal{C}^{1}$ functions between Banach spaces, Nonlinear Anal. 51 (2002), 189-195.

26. Markus, L. and Yamabe, H., Global stability criteria for differential systems, Osaka Math. J. 12 (1960), 305-317. 
27. Meyer, G., On solving nonlinear equations with a one parameter imbedding, SIAM J. Numer. Anal. 5 (1968), 7391-7752.

28. Milojevic, P. S., Implicit function theorems, approximate solvability of nonlinear equations and error estimates, J. Math. Anal. Appl. 211 (1997), 4241-7459.

29. Palais, R. S., Natural operations on differential forms, Trans. Amer. Math. Soc. 92 (1959), 125-141.

30. Pinchuk, S., A counterexample to the strong real Jacobian conjecture, Math. Z. 217 (1994), 1-4.

31. Plastock, R., Homeomorphisms between Banach spaces, Trans. Amer. Math. Soc. 200 (1974), 1691-7183.

32. Pourciau, B., On global invertibility of nonsmooth mappings, J. Math. Anal. Appl. 131 (1988), 170-179.

33. Radulescu, M. and Radulescu, S., Global inversion theorems and applications to differential equations, Nonlinear Anal. 4 (1980), 951-965.

34. Radulescu, M. and Radulescu, S., Global univalence and global inversion theorems in Banach spaces, Nonlinear Anal. 13 (1989), 5391-7553.

35. Rockafellar, R. T., Convex Analysis, Princeton Math. 28, Princeton University Press, 1970.

36. Rheinboldt, W., Local mapping relations and global implicit functions theorems, Trans. Amer. Math. Soc. 138 (1969), 1831-7198.

37. Stoilow, S., Sur les transformations continues des espaces topologiques, Bull. Math. Soc. Sci. Roumanie 35 (1933), 229-235.

38. Zampieri, G., Diffeomorphisms with Banach space domains, Nonlinear Anal. 19 (1992), 923-932.

Marcelo Montenegro

Universidade Estadual de Campinas

IMECC

Departamento de Matemática

Rua Sérgio Buarque de Holanda, 651

Campinas, SP

CEP 13083-859

Brazil

msm@ime.unicamp.br

Received February 24, 2016

in revised form March 1, 2017
Adilson E. Presoto

Universidade Federal de São Carlos

Departamento de Matemática

Rodovia Washington Luis, Km 235

São Carlos, SP

CEP 13565-905

Brazil

presoto@dm.ufscar.br 